\title{
Evaluation of tear function tests and corneal thickness in patients with meibomian gland dysfunction
}

\author{
Kuddusi Teberik ${ }^{1} \oplus$, İlter İritaş²® \\ ${ }^{1}$ Department of Ophthalmology, Düzce University School of Medicine, Düzce, Turkey \\ ${ }^{2}$ Department of Ophthalmology, Ufuk University School of Medicine, Ankara, Turkey
}

\begin{abstract}
Objectives: To investigate whether central corneal thickness (CCT) and tear function test differ from healthy controls in the ones suffering meibomian gland dysfunction (MGD).

Methods: This prospective study was carried out with 99 individuals in total (50 patients MGD, 49 healthy individuals). Schirmer-1, tear break-up time (TBUT), the Ocular Surface Disease Index (OSDI) questionnaire and CCT measurmentwas administered to all patients.

Results: The average Schirmer 1 measurements were $16.6 \pm 3.3 \mathrm{~mm}$ for right eyes, $16.4 \pm 3.6 \mathrm{~mm}$ for left eyes in the MGD group and 17.1 $\pm 3.7 \mathrm{~mm}$ for right eyes, $17.0 \pm 4.4 \mathrm{~mm}$ for left eyes in the control group. The mean TBUT values were $10.1 \pm 3.6$ seconds for right eyes, $10.2 \pm 3.4$ seconds for left eyes in the MGD group and $14.7 \pm 3.7$ seconds for right eyes, $15.8 \pm 4.1$ seconds for left eyes in the control group $(p=0.001)$. The mean OSDI score values were 40.3 \pm 23.7 in the MGD group and 19.4 \pm 8.7 in the control group $(p=0.001)$. The average CCT in the MGD group was $539.4 \pm 30.0 \mu \mathrm{m}$ and $539.7 \pm 33.0 \mu \mathrm{m}$ (right and left, respectively). The average CCT in the control group was 551.6 $\pm 32.8 \mu \mathrm{m}$ and 550.7 $\pm 32.2 \mu \mathrm{m}$ (right and left, respectively). The mean CCT measurements in the MGD group were not statistically significant compared to the healthy control group ( $p=0.059$, and $p=0.097$, right and left, respectively).

Conclusions: The Schirmer test and CCT measurements are not significantly different in patients with MGD compared to healthy control subjects.

Keywords: Meibomian gland dysfunction, Schirmer-1 test, tear break-up time, ocular surface disease index, central corneal thickness
\end{abstract}

M eibomian glands are known as a kind of modified sebaceous glands which are positioned in parallel rows inside the tarsi of the eyelids, amounting 30-40 nodes in the upper lid and 20-30 nodes in the lower lid [1], and help forming the lipid layer of the tear film to decrease aqueous tear evaporation by discharging lipids to the surface of the eye [2]. Meibomian gland dysfunction (MGD) is a chronic, common anomaly of the meibomian glands, described by terminal duct obstruction and/or qualitative/quantitative alterations in the glandular secretion. It may result in change of the tear film, indications of eye irritation, clinically significant inflammation, and ocular surface disease. The role of inflammation in the etiology of MGD is controversial and uncertain [3].

This leads to a decreased distribution of meibomian lipid to the lid margin and is connected with a changing level of lid inflammation [4]. MGD is a widespread eyelid disorder with a broad frequency of $39-50 \%$ in the US population, incidentally increasing 
with age [5].

MGD is usually clinically categorized with posterior Blepharitis; however, the terms posterior blepharitis and MGD can not be used interchangeably [3], since posterior blepharitis, as a definiton, involves significant inflammation and no significant inflammation is seen in all variations of obstructive MGD [6].

Meibomian glands, through holes located just in front of the mucocutaneous junction as a clear liquid, terminologically meibomian oil or meibum [7], synthesize and secrete a mixture of lipid. This makes the release and spread of meibum to the tear film lipid layer easier while the blink strokes upwards over the aqueous subphase. The role of tear film lipid layer is crucial in retarding the evaporation of tear fluid from the preocular tear film, so as to preventing dryness. At the same time, it helps tear film stability to improve by lowering outer tension and avoiding tear film from being contaminated by sebum [8]. Tear film instability and evaporative dry eye problems may occur due to the disorders in meibomian gland function which affect meibomian quality and quantity [9].

Chronic irritation of the ocular surface as a result of dryness is followed by immune activation and results in a decrease in film thickness, which is normally ranging from 3 to $40 \mu \mathrm{m}[10,11]$.

Assessment of central corneal thickness (CCT) informs about corneal health. CCT assessment has a clinical and practical precaution in practices such as refractive surgery, contact lens use and ocular diseases such as glaucoma and keratoconus $[12,13]$. Karadayi et al. [14] suggested using CCT assessment as a diagnosis and follow-up criteria in dry eye patients. The Meibomian gland dysfunction is one of the most common factor that cause increase of tear film evaporation. Mathers [15] demonstrated that the loss of meibomian glands decreases production of tear, and increases tear evaporation rate and tear osmolarity. It has been reported that removal of lipid layer of the tear film increases 4-fold of the evaporation of the aqueous tear [16]. In normal conditions, the aqueous tear layer film is isotonic or mildly hypotonic. Increase of tear film osmolarity and inflammation of the ocular surface are likely to be the cause of thinning of the corneal thickness [17]. Hence, in all types of dry eye, including tear deficiency and incresed evaporation, may be associated with a decreased corneal thickness.

Given the incidence of dry eye together with MGD and the possible effect of dry eye on corneal thickness measurements, we conducted a study to determine if CCT and tear function tests differed in the ones suffering from MGD compared to the ones in healthy controls.

\section{METHODS}

The prospective study groups consisted of 198 eyes:100 eyes of 50 patients diagnosed with MGD aged between 18 and 65 years and 98 eyes of 49 healthy individuals (control group) aged between 18 and 60 years without any ophthalmic or systemic pathology following their application to our outpatient for routine examination. Written consent was obtained from all patients before the study, conducted according to ethical committee guidelines and accordingly to the principals of the Helsinki Declaration.

Patients with a history of ophthalmological or systemic pathology or who used topical medication during the study were excluded from the study. For each participant, a full ophthalmological examination including refraction, external eye examination, slitlamp examination and evaluation of posterior segments was performed.

In addition to subjective complaints in the diagnosis of MGD, biomicroscopic examination depended on the existence of all of the following: frothy tear accumulation on lower eyelid edge; shortening of tear break up time; redness in tarsal and bulbar conjunctiva; papillary reaction on the tarsus; irregularity at the eyelid margin; telangiectatic vessel formation; and inclusion in the mouth of the meibomian glands (plugging) [9].

Along with the common check-up, first tear breakup time, then Schirmer-1 was measured. The tear break-up time (TBUT) was evaluated by applying one drop of $2 \%$ sodium fluorescein dye into the eye without anesthesia. After the patient had been blinded three to four times, the tear film was observed using a biomicroscope with a broad light blue cobalt filter. The time to the first break in the tear film complex after the last blink was measured. The Schirmer 1 test was detected by putting aSchirmer strip into the temporal inferior fornices. Topical anesthesia was not preferred. The outcomes were obtained as the number of millimeters of wetting at five minutes. 
Table 1. Comparison of CCT and dry eye tests between groups

\begin{tabular}{lcccccc}
\hline Parameters & \multicolumn{2}{c}{ MGD group } & \multicolumn{2}{c}{ Control group } & \multicolumn{2}{c}{ p value } \\
& R & L & R & L & R & L \\
\hline CCT $(\mu \mathrm{m})$ & $539.4 \pm 30.0$ & $539.7 \pm 33.0$ & $551.6 \pm 32.8$ & $550.7 \pm 32.2$ & 0.059 & 0.097 \\
Schirmer 1 $(\mathrm{mm})$ & $16.6 \pm 3.3$ & $16.4 \pm 3.6$ & $17.1 \pm 3.7$ & $17.0 \pm 4.4$ & $\mathbf{0 . 0 0 1}$ & $\mathbf{0 . 0 0 1}$ \\
BUT $(\mathrm{sec})$ & $10.1 \pm 3.6$ & $10.2 \pm 3.4$ & $14.7 \pm 3.7$ & $15.8 \pm 4.1$ & $\mathbf{0 . 0 0 1}$ & $\mathbf{0 . 0 0 1}$ \\
OSDI & \multicolumn{2}{c}{$40.3 \pm 23.7$} & \multicolumn{2}{c}{$19.4 \pm 8.7$} & $\mathbf{0 . 0 0 1}$ \\
\hline Data are shown as mean \pm standard deviation. CCT $=$ central corneal thickness, TBUT $=$ the tear break up time,
\end{tabular}

Data are shown as mean \pm standard deviation. CCT $=$ central corneal thickness, TBUT $=$ the tear break up time, OSDI = Ocular Surface Disease Index, $\mathrm{R}=$ right eye, $\mathrm{L}=$ left eye

The Ocular Surface Disease Index (OSDI) questionnaire was administered to all patients. The OSDI questionnaire, consisting of 12 questions, aims to question the patient's complaints about the dry eye in the last two weeks, and scales the severity of the disease by scoring the effects of these complaints on daily activities, environmental causes, duration of the complaints and the severity, along with the complaints of the patient [18].

Finally, the CCT was measured using an ultrasonic pachymetry (Echoscan US 500 Nidek Co. Ltd, Aichi, Japan). Topical proparaine hydrochloride was used as anesthesia in the cornea. Measurements were taken as the point of the probe was targeted to the center of the pupil and was vertical to the cornea while the subject was looking at a planned spot. Three consecutive measurements were taken at the center of the cornea of each eye and the average value was measured. A 20-minute period was applied between all measurements.

\section{Statistical Analysis}

The measurements were described as the mean and standard deviation. The normality of the data was assessed using the Shapiro-Wilk test and the difference between the two groups using Student t-tests. Pearson correlation test was performed for the correlation between variables. A value of $p<0.05$ was considered statistically significant.

\section{RESULTS}

While mean age was $42.0 \pm 13.8$ years in the MGD group, mean age level was found as $41.2 \pm 11.2$ years among the controls $(p=0.642)$. The best corrected visual acuity of all patients in both groups was 1.00 according to LogMAR. While the female to male ratio was $1.7 / 1$ in the MGD group, the ratio was found as $2.5 / 1$ the controls. There was no statistically significant difference between groups with respect to sex comparison $(p=0.429)$. The mean Schirmer 1 values were $16.6 \pm 3.3 \mathrm{~mm}$ for right eyes, $16.4 \pm 3.6$ $\mathrm{mm}$ for left eyes in the MGD group and $17.1 \pm 3.7 \mathrm{~mm}$ for right eyes, $17.0 \pm 4.4 \mathrm{~mm}$ for left eyes in the control group ( $p=0.894$, and $\mathrm{p}=0.365$, right and left, respectively). The mean TBUT values were $10.1 \pm 3.6$ seconds for right eyes, $10.2 \pm 3.4$ second for left eyes in the MGD group and $14.7 \pm 3.7$ seconds for right eyes, $15.8 \pm 4.1$ seconds for left eyes in the control

Table 2. Correlation coefficients between CCT, Schirmer 1, TBUT and OSDI

\begin{tabular}{lllccccc}
\hline & & \multicolumn{2}{c}{ Schirmer 1 } & \multicolumn{2}{c}{ TBUT } & \multicolumn{2}{c}{ OSDI } \\
& $\mathbf{R}$ & $\mathbf{L}$ & $\mathbf{R}$ & $\mathbf{L}$ & $\mathbf{R}$ & $\mathbf{L}$ \\
\hline CCT & $\boldsymbol{p}$ value & 0.411 & 0.273 & 0.515 & 0.692 & 0.314 & 0.223 \\
& $\mathbf{r}$ & -0.084 & -0.111 & -0.066 & -0.040 & 0.102 & 0.121 \\
\hline
\end{tabular}

Data are shown as mean \pm standard deviation. $\mathrm{CCT}=$ central corneal thickness, TBUT $=$ the tear break up time, OSDI = Ocular Surface Disease Index, $\mathrm{R}=$ right eye, $\mathrm{L}=$ left eye 
group $(\mathrm{p}=0.001)$. The mean OSDI score values were $40.3 \pm 23.7$ in the MGD group and $19.4 \pm 8.7$ in the control group $(p=0.001)$.

The average CCT in the MGD group was $539.4 \pm$ $30.0 \mu \mathrm{m}$ and $539.7 \pm 33.0 \mu \mathrm{m}$ (right and left, respectively). The average CCT in the control group was $551.6 \pm 32.8 \mu \mathrm{m}$ and $550.7 \pm 32.2 \mu \mathrm{m}$ (right and left, respectively). The mean CCT measurements in the MGD group were not statistically significant compared to the healthy control group ( $p=0.059$, and $p=0.097$, right and left, respectively) (Table 1).

There was no statistically significant correlation between CCT, Schirmer 1, TBUT and OSDI tests in the eyes MGD $(p>0.05)$ (Table 2).

\section{DISCUSSION}

MGD is one of the most frequently encountered disorders in ophthalmic practice. Bron and Tiffany [4] reported that $38.9 \%$ of the patients admitted to the routine ophthalmic examination suffer from MGD. Meibomian gland dysfunction is a chronic disease that results in decreased lipid secretion to the ocular surface due to occlusion of the meibomius gland. People with MGD usually suffer from notable obstacles, such as burning, itching, irritation, and photophobia. They might have a cloudy vision, contact lens intolerance, ocular surface damage and ocular infection. The use of contact lenses can aggravate symptoms of MGD even if the contact lens intolerance does not ocur $[19,20]$.

This study shows that the mean CCT and Schirmer 1 test was not significantly different from normal eyes in patients with MGD with dry eyes. However, according to the control group, it was found that there was thinning of the central corneal thickness more than 10 microns. There was a statistically significant difference between the control group and the MGD group in terms of the mean values of TBUT and OSDI scores.

Akyol-Salman et al. [21] assessed CCT values in patients with MGD and in healthy individuals. They also did not find significant differences between the groups [21]. Ultrasonic pachymeter (UP) devices are still accepted as the gold standard technique and commonly used because of the easiness of its handling, portability and rapid measurement time [22].
In our study, an ultrasonic pachymeter was used for CCT measurement like Akyol-Salman et al. [21]. Yuksel et al. [23] found a statistically significant decline in CCT as a result of MGD in the 6-month treatment of patients treated with systemic isotretinoin for acne vulgaris. Meibomian glands discharge lipids into the tear film and create a superficial lipid layer stabilizing it. MGD is identified by terminal duct blockage or glandular secretion changes and immediately afterwards, tear evaporates, tear osmolarity increases, aqueous tear volume declines, and inflammation cycle begins [4, 24]. Additionally, apoptosis in the ocular surface tissues, including the central and peripheral corneal epithelial, was shown in an experimental dry-eye study in mice[10]. SanchisGimeno et al. [25] and Liu and Pflugfelder [27] found a statistically significant decrease in corneal thickness values in dry eyes. Pole and Batzer [11] observed in their studies that there was a reduced corneal thickness in dry eyes, yet dry and normal eyes did not significantly differ from each other. Likewise, Van Bijsterveld and Baardman [27] obtained compared mean CCT values in patients with keratoconjunctivitis sicca and healthy control subjects. We can say that the differences between the CCT measurement values in the studies in which the dry eye and the CCT relationship in the literature are studied are derived from the measurement methods. However, we believe that these measurements should be repeated in a wider population with newdevices developed to measure CCT.

In our study, all patients with MGD had various symptoms and specific lid changes. Despite TBUT shortening due to dry eye as a result of excessive evaporation, the Schirmer test results were normal due to the absence of aqueous insufficiency. Demircan et al. [28] found that Schirmer test results were similar to healthy subjects and that TBUT was significantly shorter in the study of patients with chronic blepharitis. Sayman Muslubaş et al. [29] showed increased score in OSDI questionnaire and shortening in TBUT without any decrease in the Schirmer test measurements in their studies comparing healthy individuals with MGD patients. Taşkıran Cömez et al. [18] found, in a study comparing 40 patients with dry eye syndrome and 39 healthy subjects, that the OSDI score average was statistically significantly higher in the patients with dry eye syndrome. Similarly, in our 
study, OSDI score was found to be higher in the MGD group which caused dry eyes due to evaporation increase. We also examined whether there was a correlation between CCT and Schirmer 1, TBUT, OSDI score in our study, but no significant correlation was found between these parameters.

The weakness of our study is not being able to measure tear osmolarities and apply non-contact meibography. The strength of our study is that more patients are included in the study than the number of patients in the literature. In addition, the correlation between CCT and Schirmer 1, TBUT and OSDI score, which had not been observed before, has been examined in our study.

\section{CONCLUSION}

As a result, dry eye syndrome may occur depending on the MGD, as is the cause of the MGD. High score in OSDI questionnaire, the fact that Schirmer test measurements are not different from healthy individuals and shortening of TBUT in MGD support the diagnosis. For this reason, using TBUT and OSDI questionnaires will be appropriate to determine the presence of a dry eye in such cases. In addition, the CCT analyzes are not significantly different between patients with MGD and healthy control subjects.

\section{Conflict of interest}

The authors disclosed no conflict of interest during the preparation or publication of this manuscript.

\section{Financing}

The authors disclosed that they did not receive any grant during conduction or writing of this study.

\section{REFERENCES}

1. Greiner JV, Glonek T, Korb DR, Whalen AC, Hebert E, Hearn $\mathrm{SL}$, et al. Volume of the human and rabbit meibomian gland system. In: Lacrimal Gland, Tear Film, and Dry Eye Syndromes 2. Springer, Boston: MA; 1998: pp. 339-43.

2. Mishima S, Maurice DM. The oily layer of the tear film and evaporation from the corneal surface. Exp Eye Res 1961;1:3945

3. Nichols KK, Foulks GN, Bron AJ, Glasgow BJ, Dogru M,
Tsubota K, et al. The international workshop on meibomian gland dysfunction: executive summary. Invest Ophthalmol Vis Sci 2011;52:1922-9.

4. Bron AJ, Tiffany JM. The contribution of meibomian disease to dry eye. Ocul Surf 2004;2:149-65.

5. Lemp MA, Nichols KK. Blepharitis in the United States, a survey-based perspective on prevalence and treatment. Ocul Surf 2009;7:1-14.

6. Blackie CA, Korb DR, Knop E, Bedi R, Knop N, Holland EJ. Nonobvious obstructive meibomian gland dysfunction. Cornea 2010;29:1333-45.

7. Nicolaides N, Kaitaranta JK, Rawdah TN, Macy JI, Boswell FM 3rd, Smith RE. Meibomian gland studies: comparison of steer and human lipids. Invest Ophthalmol Vis Sci 1981;20:52236.

8. Bron AJ, Benjamin L, Snibson GR. Meibomian gland disease. Classification and grading of lid changes. Eye (Lond) 1991;5:395-411.

9. Driver PJ, Lemp MA. Meibomian gland dysfunction. Surv Ophthalmol 1996;40: 343-67.

10. Yeh S, Song XJ, Farley W, Li DQ, Stern ME, Pflugfelder SC. Apoptosis of ocular surface cells in experimentally induced dry eye. Invest Ophthalmol Vis Sci 2003;44:124-9.

11. Pole JJ, Batzer JK. Central corneal thickness of patients with dry eyes. J Am Optom Assoc 1985;56:220-1.

12. Doughty MJ, Zaman ML. Human corneal thickness and its impact on intraocular pressure measures: a review and metaanalysis approach. Surv Ophthalmol 2000;44:367-408.

13. deSanctis U, Missolungi A, Mutani B, Richiardi L, Grignolo FM. Reproducibility and repeatability of central corneal thickness measurement in keratoconus using the rotating Scheimpflug camera and ultrasound pachymetry. Am J Ophthalmol 2007;144:712-8.

14. Karadayi K, Ciftci F, Akin T, Bilge AH. Increase in central corneal thickness in dry and normal eyes with application of artificial tears: a new diagnostic and follow-up criterion for dry eye. Ophthalmic Physiol Opt 2005;25:485-91.

15. Mathers WD. Ocular evaporation in meibomian gland dysfunction and dry eye. Ophthalmology 1993;100:347-51.

16. Craig JP, Tomlinson A. Importance of the lipid layer in human tear film stability and evaporation. Optom Vis Sci 1997;74:8-13. 17. Dayanir V, Sakarya R, Ozcura F, Kir E, Aktunç T, Ozkan BS, et al. Effect of corneal drying on central corneal thickness. J Glaucoma 2004;13:6-8.

18. Taşkıran Cömez A, Tufan HA, Kocabıyık O, Gencer B. Effects of lubricating agents with different osmolalities on tear osmolarity and other tear function tests in patients with dry eye. Curr Eye Res 2013;38:1095-103.

19. Korb DR, Hendriquez AS. Meibomian gland dysfunction and contact lens intolerance. J Am Optom Assoc 1980;51:243-51.

20. Paugh JR, Knapp LL, Martinson JR, Hom MM. Meibomian therapy in problematic contact lens wear. Optom Vis Sci 1990;67:803-6.

21. Akyol-Salman I, Azisi S, Mumcu U, Öndaş O, Baykal O. Central corneal thickness in patients with meibomian gland dysfunction. Clin Exp Optom 2011;94:464-7.

22. Lee YG, Kim JH, Kim NR, Kim CY, Lee ES. Comparison 
between tonopachy and other tonometric and pachymetric devices. Optom Vis Sci 2011;88:843-9.

23. Yuksel N, Ozer MD, Akcay E, Ozen U, Uzun S. Reduced central corneal thickness in patients with isotretinoin treatment. Cutan Ocul Toxicol 2015;34:318-21.

24. Jackson WB.Blepharitis: current strategies for diagnosis and management. Can J Ophthalmol 2008;43:170-9.

25. Sanchis-Gimeno JA, Lleo'-Pe'rez A, Alonso L, Rahhal MS, Martínez-Soriano F. Reduced corneal thickness values in postmenopausal women with dry eye. Cornea 2005;24:39-44.

26. Liu Z, Pflugfelder SC. Corneal thickness is reduced in dry eye. Cornea 1999;18:403-7.

27. van Bijsterveld OP, Baardman J. [Measurements of corneal thickness in patients with keratoconjunctivitis sicca]. Klin Monbl Augenheilkd 1990;197:240-3. [Article in German]

28. Demircan A, Altan Ç, Azman FE, Alkin Z, Küçüksümer Y, Demirok A. [Tear properties in eyes with chronic blepharitis]. Turk J Ophthalmol 2012;42:346-348. [Article in Turkish]

29. Sayman Muslubaş IB, Kahya A, Özertürk Y. [Tear function test results in patients with meibomian gland dysfunction]. J Kartal TR 2014;25:23-6. [Article in Turkish] 